\title{
Thermophysiological responses to capacitive resistive monopolar radiofrequency electromagnetic radiation in patients with osteoarthritis of the knee joint: a randomised controlled experimental study.
}

Authors: Kumaran, B; Watson, T

\begin{abstract}
This study investigated the effect of $448 \mathrm{kHz}$ capacitive resistive monopolar radiofrequency (CRMRF) on the superficial and deep physiological responses of patients with osteoarthritis (OA) of the knee(s). Forty-five patients diagnosed with OA in their knee(s) were enrolled into a three-group randomised controlled study, from the waiting list of a local hospital. They received localised treatment with either CRMRF, CRMRF placebo or a control (no treatment) to the knee for 15 minutes. Pre, post and 20-minute follow-up measurements of skin temperature (SKT) and skin blood flow $(\mathrm{SBF})$ were obtained from the knee using the FlexComp Infiniti (SA7550) physiological measurement system. Pre and post treatment deep blood flow were recorded using Doppler ultrasound. Core temperature, blood pressure (BP) and pulse rate (PR) were concurrently monitored. Group data were compared using the ANOVA model. Statistical significance was set at $\mathrm{p} \leq 0.05,0.8$ power, and $95 \% \mathrm{CI}$. Significant increases and sustenance of SKT and SBF, and significant increases in volume and intensity of deep blood flow were demonstrated with CRMRF over the placebo and control interventions in all comparisons $(p<0.001)$. No meaningful changes in blood flow velocity, core temperature, BP or PR were noted for any condition. The findings were markedly more pronounced than those previously reported in asymptomatic adults. However, the patients had received a higher average dose of CRMRF (mean (SD): 46.87 (4.08) W) compared to the asymptomatic sample (mean (SD): 42.37 (4.64) W), therefore further
\end{abstract}


research is needed to better understand the differences in physiological responses between patients and asymptomatic people.

\section{Key words}

Electrophysical agents; Radiofrequency treatment; Osteoarthritis of knee; Blood flow; Skin temperature.

\section{Introduction}

Radiofrequency (RF)-based electrophysical agents (EPA) are used in therapy to reduce pain and inflammation and promote tissue healing. There is a growing body of evidence from clinical studies and systematic reviews to support their use (Kumaran \& Watson, 2019; Laufer \& Dar, 2012; Wang et al., 2017; Yilmaz Kaysin et al., 2018). Devices that employ much lower frequencies of RF when compared to the most commonly used shortwave therapies (SWT, frequency typically at $27.12 \mathrm{MHz}$ ), are becoming increasingly available. Capacitive resistive monopolar radiofrequency (CRMRF) is a low frequency RF that operates at $448 \mathrm{kHz}$. Although this mode of therapy is relatively new, it is used by clinicians worldwide as a safe form of RF-based therapy. Research into the physiological and clinical effects of such low frequency RF (RF below the shortwave frequencies) has gained momentum particularly in the last decade with increasing number of studies being reported. Recently, the same authors published several studies on the effects of CRMRF in asymptomatic people as well as in patients (Kumaran et al., 2017; Kumaran \& Watson, 2015, 2018, 2019). All these studies reported significant physiological responses such as a sustained rise in skin temperature (SKT), skin blood flow (SBF) and deep blood flow in an asymptomatic sample in addition to clinical benefits such as improvement in pain and function among patients affected by osteoarthritis (OA) of the knee joint. Nonetheless, the overall evidence base for RF-based EPA below the shortwave frequencies remains suboptimal. 
Several thermophysiological effects are induced by the absorption of RF energy into the tissues (Adair, 1995; Foster \& Glaser, 2007; Foster \& Morrissey, 2011; Silverman \& Pendleton, 1968). At relatively high doses, the effects of RF are predominantly thermal (AlMandeel \& Watson, 2010; Bricknell \& Watson, 1995; Draper et al., 1999; Valtonen et al., 1973). The conversion of RF energy into heat energy in tissues and the ensuing thermophysiological responses can lead to various physiological and therapeutic benefits. A modest rise in temperature (mild hyperthermia) is enough to accelerate and/or increase cellular metabolic activity, and heat-induced vasodilatation can enhance local blood circulation in the tissues (Adair \& Black, 2003; Challis, 2005; Jauchem, 2008; Silverman \& Pendleton, 1968). Heat can also reduce muscle tone and improve tissue extensibility depending on the level of temperature rise attained in the tissues (Draper et al., 2004; Petrofsky et al., 2013; Robertson et al., 2005). However, it can be questioned whether these physiological responses translate to clinical benefits.

The current study addresses the above question to some extent as the included data was collected from the same clinical trial that aimed to evaluate the short to medium term effect of CRMRF on the symptoms and function in patients with OA knee, the results of which have already been published (Kumaran \& Watson, 2019). The same patients participated in this physiological component of the study on their first visit to the hospital. The clinical study found that the treatment of OA with a moderately thermal dose of CRMRF produced clinically significant improvements in pain and functional quality of life in the short term, which was significantly more pronounced than those obtained with a placebo or current standard treatment for OA. Separately, this paper also enables a comparison of the patient physiological data with the previously reported normative physiological data (Kumaran et al., 2017; Kumaran \& Watson, 2015, 2018). 


\section{Materials and methods}

\section{Apparatus}

\section{CRMRF device}

The CRMRF energy at $448 \mathrm{kHz}$ was delivered using the commercially available 'Indiba Activ 902' (Indiba S. A., Barcelona). This CE marked therapeutic device was factory calibrated and pretested for accuracy of output. The peak power of the device is $200 \mathrm{~W}$ (450 VA (Volt-Ampere)). It delivers continuous-wave radiofrequency energy in two modes: Capacitive (CAP) and Resistive (RES), using metallic electrodes. The CAP electrode is coated with a polyamide material that acts as a dielectric medium. This medium insulates its metallic body from the skin surface, thereby forming a capacitor with the treated tissues. The RES electrode is uncoated. Energy delivery to the body is enabled using a conductive cream (coupling medium), since the frequency of the RF is not high enough to be able to conduct through air. Electrodes measuring $65 \mathrm{~mm}$ in diameter were used for treatment and a flexible rectangular metallic electrode measuring 200 × $260 \mathrm{~mm}$ was used as return electrode throughout the current study.

\section{The FlexComp Infiniti system}

The FlexComp Infiniti (SA7550) (Thought Technology Ltd., Montreal, Canada) was used to record SKT and SBF. It is a 10-channel multi-modality encoder for real-time computerized psychophysiology, biofeedback and data acquisition. It is a portable unit that can render a wide range of objective physiological measures in clinical practice and research. The device has been used in research in other subject areas (Mandryk \& Atkins, 2007; Mandryk et al., 2006; Noe et al., 2009). 


\section{Doppler ultrasound}

Blood flow to the deeper tissues (two centimetres or more from the skin) was monitored using a portable Doppler ultrasound that provided information on the velocity, volume and intensity of blood flow. An 'Esaote MyLab25' (Esaote S.p.A, Genoa, Italy) ultrasound scanner was used alongside a linear array probe 'LA523' that supports a frequency range of 4-13 MHz (Esaote S.p.A, Genoa, Italy) to collect the data.

\section{Other devices}

Blood pressure $(\mathrm{BP})$ and pulse rate $(\mathrm{PR})$ were monitored using a digital $\mathrm{BP}$ monitor (Omron M2, Omron Healthcare Europe B.V., Netherlands) and core temperature was measured using an infra-red (IR) tympanic thermometer (Braun ThermoScan IRT 4520, Braun GmbH, Germany). A body composition monitor (Omron BF508, Omron Healthcare Europe B.V., Netherlands) was used to obtain the anthropometric data.

\section{Study design and setting}

A placebo-controlled participant-blinded randomised controlled trial was conducted to address the aim of the study. The study was carried out at the outpatient physiotherapy wing of the Safari Therapy Unit in the Hemel Hempstead General Hospital, Hemel Hempstead (Hertfordshire Community NHS Trust). The ethics approval for the study was granted by the 'NRES Committee North West - Greater Manchester South' under the Research Ethics Service of the NHS Health Research Authority (HRA) (REC reference: 15/NW/0529; Protocol number: HSK/PG/NHS/00312; IRAS project ID: 173691). The study was registered with the National Institute of Health Research (NIHR) Clinical Research Network (CRN) (Portfolio) (study ID: 20264). 


\section{Sample and groups}

The study involved three groups: experimental, sham and control. Forty-five patients diagnosed with OA knee meeting the American College of Rheumatology criteria (Altman et al., 1986), and symptomatic for a minimum of six months, were recruited if they were on the waiting list for physiotherapy treatment. They were randomly allocated into an experimental (CRMRF), sham (CRMRF placebo) and control (no treatment) groups with 15 participants in each group, using a computer-generated randomisation chart (IBM SPSS Statistics, Version 20), and blinded from the participants using allocation concealment. People with known contra-indications to RF-based treatment (e.g. pregnancy, active cancer, metal implant in the affected knee, cardiac pacemaker), or those with significant comorbidities (e.g. neurological impairment, open skin wounds in the area) were excluded.

\section{Interventions}

\section{CRMRF}

A moderately thermal dose of CRMRF was delivered to the experimental group participants for 15 minutes. The intensity of delivery was gradually increased till the participants reported moderate yet comfortable heating, which was then maintained throughout the session. Fifteen minutes of treatment comprising five minutes CAP and 10 minutes RES was delivered to the whole knee joint area using $20 \mathrm{ml}$ coupling medium for each mode. The return electrode was placed under the same limb one-fourth way down the distance between the fibular head and the lateral malleolus.

\section{CRMRF placebo}

An additional placebo setting was integrated into the device by the manufacturer for the sham group in this study. The treatment duration and technique were identical to the active intervention except that no verbal feedback was sought from the participants as there was no 
energy delivery. The machine display was indistinguishable between real and placebo modes. However, the operator could not be blinded to the interventions due to the thermal perception that occurs during the real CRMRF treatment.

\section{Control}

In the control group the participants rested on the treatment plinth for the duration of the intervention receiving no treatment.

\section{Procedure}

The participants attended the study by avoiding food, beverages and strenuous exercises within one hour before the start of their session. This was to ensure that their physiological condition remained stable during the sessions (Al-Mandeel \& Watson, 2010; Drust et al., 2003; Jackson \& Pollock, 1978). After screening and signing of the informed consent, demographic and anthropometric data were collected. Subsequently the participants changed to own comfortable loose clothing and positioned themselves in supine fully supported using pillows on a plinth. They were allowed to acclimatize to the room conditions for 20 minutes. Skin over the medial aspect of the affected knee joint was prepared and marked. Skin temperature was recorded from the middle of the medial joint line every two minutes until it stabilised (baseline skin temperature). The core (tympanic) temperature was also monitored. The room temperature was recorded at the beginning and end of all sessions, but it was not possible to be controlled. The experimental timeline and measurements are illustrated in figure 1.

\section{Skin surface data}

All skin physiological measurements of temperature (SKT) and blood flow (SBF) were performed pre-treatment, immediately post treatment and 20-minute post treatment for all three groups. The PPG and SKT probes from the FlexComp Infiniti data acquisition system 
were attached to the skin on marked areas on the medial joint line. The probes were positioned within the treatment zone but were removed prior to the RF treatment and reattached post treatment. Reliability of probe placement was established by extensive pilot work. The sampling rate, and the sampling duration at each measurement point were pre-set by the manufacturer at 256 samples per second and 80 seconds respectively. The core temperature, BP and PR were also concurrently monitored.

\section{Deep blood flow data}

The deep blood flow measurements were performed twice, pre and post intervention. For each participant, the most prominent pulsatile (arterial) blood flow was identified over the lower anteromedial aspect of the quadriceps femoris muscle. Skin markings were used to establish the accuracy of probe placement and ensure repeatability. Prior to this study, the intra-rater reliability for Doppler measurements was established in a separate pilot study. The pre and post measurement settings remained consistent throughout the study. Colour Doppler recordings for velocity of flow were performed first followed by power Doppler for volume and intensity of flow. Up to five seconds of blood flow data were recorded during each measurement, which were then broken down into image frames for analysis.

\section{Data analysis}

All data were analysed using Microsoft Office Excel 365 and IBM SPSS Statistics Version 20 (IBM Corporation). To examine the statistically significant differences within or between the groups, group data were compared using a mixed model analysis of variance (ANOVA) at three within-group time points (baseline, post treatment and follow-up) and three betweengroup conditions (experimental, placebo and control). Where the data deviated from normality and/or showed inequality of variances, data transformation was performed using the square root function. Separately, in order to enable a comparison between the patient data 
and the previously published normative data (Kumaran et al., 2017; Kumaran \& Watson, 2018), percentage changes of means were plotted. Since the two sets of data were not directly comparable, data normalization was carried out by taking the baseline scores as 'zero' and the 'percentage changes from baseline' as the subsequent points. The two sets of data were compared based on their face validity as the equivalence between the two sets of devices (Biopac and FlexComp, and the two ultrasound devices) was not tested in this study. The statistical significance was set at $\mathrm{p} \leq 0.05(0.8 \mathrm{P}, 95 \% \mathrm{CI})$.

\section{Processing of image data}

Prior to statistical analysis, ultrasound images were computationally analysed using MATLAB (MathWorks, Massachusetts) algorithms to process the colour image data into numerical data. For every image frame the MATLAB algorithms generated two types of data: the 'pixel count' (total number of coloured pixels expressed in 'kilo pixels') in the 'region of interest' (ROI) indicating the blood volume information, and the 'colour intensity index' (sum of colour intensity values of all the coloured pixels in the ROI expressed in 'kilo pixels'). In the final analysis, the colour intensity index as the 'mean colour intensity index per pixel' by dividing the sum of colour intensity values of a frame by the total number of pixels in that frame. In colour Doppler, the 'colour intensity index' indicated the relative velocity of blood flow, where higher the index higher was the velocity. In power Doppler, the pixel count represented the overall 'blood volume' and the colour intensity index represented the 'blood flow intensity'. Higher the pixel count and/or index, higher was the blood flow. The analysis of all machine colour scales using MATLAB has been reported earlier (Kumaran et al., 2017).

\section{Results}

All participants completed the study. All procedures were well tolerated and there were no reports of any adverse events. Figure 2 shows sample data obtained from one of the study 
participants in the CRMRF group. The demographic and the mean (SD) anthropometric data from the participants are reported in table 1 . There were no significant differences between any of the three groups in either characteristic. The mean (SD) CAP, RES and total dose of CRMRF received by the participants in the experimental group were $17.1(2.3) \mathrm{W}, 61.7$ (6.3) $\mathrm{W}$ and 46.9 (4.1) W respectively. The energy delivered to the sham group was zero.

The results of each physiological parameter obtained from the study groups are reported below. There was no significant difference in the baseline values of any of the physiological parameters between the three groups.

\section{Skin physiological responses}

Figures $3 \& 4$ show the mean (SD) SKT and SBF recorded at the three time points and their percentage changes of mean. The statistical analysis $(3 * 3$ mixed methods ANOVA (time, group)) revealed a highly significant main effect for time (within-group change) and a highly significant interaction between group and time. Therefore, the type of intervention made a significant difference to the reported SKT and SBF values and there was a significant overall difference between the pre, post and 20-minute follow-up response (table 2).

Results of the pairwise comparisons that show changes within groups over time and the differences between groups (adjustment for multiple comparison: Bonferroni), their significance values and effect sizes (where the interaction was relevant and meaningful) are reported in tables $3 \& 4$. All significant effects and differences have been highlighted.

Overall, it is evident that the experimental group differed significantly from both placebo and control groups. The difference in SKT between the placebo and control groups is likely due to the drop in SKT in the placebo group due to the cold nature of the coupling medium. 


\section{Deep blood flow responses}

Figures $5 \& 6$ show the mean (SD) deep blood flow volume and intensity of flow recorded using power Doppler ultrasound pre and post treatment and their percentage changes of mean. The statistical analysis $(2 * 3$ mixed methods ANOVA (time, group)) revealed a highly significant main effect for time (within-group change) and a highly significant interaction between group and time. Therefore, the type of intervention made a significant difference to the reported blood flow volumes and intensities of flow and there was a significant overall difference between the pre and post results (table 2). The blood flow velocity results were unremarkable. The statistical analysis of the blood flow velocity data $(2 * 3$ mixed methods ANOVA (time, group)) did not reveal any significant main effect for time (within-group change) although there was a small yet significant interaction between group and time (table 2).

Results of the pairwise comparisons that show changes within groups over time and the differences between groups (adjustment for multiple comparison: Bonferroni), their significance values and effect sizes (where the interaction was relevant and meaningful) are reported in tables $3 \& 4$. All significant effects and differences have been highlighted. Like SBF, any change in deep blood flow responses over time was only noted within the experimental group, which was also significantly higher than that of the other two groups.

\section{Other results}

No significant variations were noted in any of the groups for core temperature, PR or BP at any point.

\section{Comparison between the normative and patient data}

As stated, data relating to the normative (asymptomatic) sample have already been published (Kumaran et al., 2017; Kumaran \& Watson, 2018). Figures $7 \& 8$ compare the percentage 
changes from baseline for each type of outcome obtained from the normative sample to that of the patient data obtained from the current study. Given these are percentage differences and given the disparities in the actual treatment dosages and methodologies between the two studies, a statistical comparison is not attempted. Nonetheless, the graphical comparison shows that the normative population responded differently to the patient population at time points two (post treatment) and three (20-minute follow-up) for all outcomes. The responses were markedly more pronounced among the patients. Some of the potential reasons for these differences in response are discussed below.

\section{Discussion}

This paper reports the results of a study on the physiological effects of CRMRF in adult patients affected by chronic OA of the knee joint. As stated, the physiological testing was carried out concomitantly with the clinical RCT on the same study group that examined the benefits of a four-week CRMRF treatment programme on pain and function caused by OA knee. In addition, this paper enabled a comparison of the current data with the previously reported study that had demonstrated similar results in asymptomatic adults (Kumaran et al., 2017; Kumaran \& Watson, 2018). However, all five experimental groups from the laboratory study were not replicated in the patient physiological study owing to time constraints and the fact that priority was given to the concomitant clinical outcomes study that involved only three groups.

To the authors' knowledge no similar physiological studies conducted on patients employing RF below the shortwave frequency range have been reported so far. A limited number of such studies employing SWT (Al-Mandeel, 2004; Guirro et al., 2015; Teslim et al., 2013) are available. The authors are therefore unable to draw any direct comparisons from the literature. In a study on the physiological effect of pulsed SWT (PSWT) on patients with OA knee, Al-Mandeel (2004) reported similar higher-level skin physiological responses such as 
SKT and SBF among the patients when compared to those obtained from asymptomatic adults. Similar doses of PSWT were used for both groups of participants. The study also suggested that in either group of participants, the key factor that may have determined the extent of physiological effect might be the applied dose of RF, with a significantly low dose of PSWT proving only as good as a placebo (Al-Mandeel, 2004).

From the results of the current study it is evident that the physiological responses obtained from patients were substantially more pronounced than those obtained from asymptomatic adults; however, the patients received a significantly higher average treatment dose $(p=$ 0.013) in comparison to the earlier normative study. The mean (SD) CRMRF doses delivered in the normative study and the patient study were 42.37 (4.64) W and 46.87 (4.08) W respectively, which means the mean dose (and hence the total energy delivered) was about $10 \%$ higher in the patient study. While the percentage change in SKT after CRMRF intervention was commensurately higher among patients, the skin and deep blood flow responses (except blood flow velocity) were more than twice greater among the patients. However, in agreement with the normative study, no significant changes were noted in the core temperature, BP or PR in any patient group at any time.

Although the RF treatment doses and the resultant physiological responses were different between the two studies, the whole-body 'specific absorption rate' (SAR, which is a measure of the rate at which energy is absorbed per unit mass by a human body when exposed to an RF field) of RF among the two samples were not significantly different from each other, effectively negating the effects of a difference in the overall dose. The mean (SD) whole body SAR was estimated to be $0.54(0.08) \mathrm{W} / \mathrm{kg}$ in the patients and $0.60(0.09) \mathrm{W} / \mathrm{kg}$ in the normative sample (SAR marginally lower in patients). However, the authors appreciate that the concept of 'whole-body SAR' is less informative here due to the localised nature of the exposures/absorption. It could be argued that the RF was completely absorbed in a defined 
mass of tissue, which in this case is the knee joint and hence a more accurate localised SAR should be reported. This required an accurate estimation of the mass of the treated knee joint area. Such more complex estimation using mathematical modelling could be problematic for arthritic joints (especially when swelling is present) and was outwith the scope of this project given the predominant clinical nature of the original study.

One of the differences between the normative study and the patient study was that the laboratory provided a more controlled environment in terms of room temperature, humidity and any potential noise due to interference from other RF fields. The room temperature was always monitored during the normative study and maintained within the thermoneutral zone for humans. However, it was not possible to alter the room temperature in the hospital although it was recorded at the beginning and end of each session. Also, there was no provision to monitor the humidity levels in the hospital treatment room. The mean (SD) room temperatures for the CRMRF groups in the normative and patient study were $25.12(1.14){ }^{\circ} \mathrm{C}$ and $24.27(0.93){ }^{\circ} \mathrm{C}$ respectively. These were not significantly different. Besides, there was no indication from either study that room temperature and/or humidity correlated significantly with the physiological responses.

The current study had its limitations like the normative study. There was no blinding on the part of the therapist who delivered the interventions and performed the assessments making this study only single-blind at best. The operator could not be blinded to the two CRMRF interventions due to the thermal perception that occurs during the real CRMRF treatment. The post treatment skin measurements could only be started with a delay of a minimum of two minutes after the end of treatment due to skin preparation time. The start of deep blood flow measurements was delayed even further, by at least five minutes after the end of treatment. Further, like the laboratory study, temperature from the deeper tissues were not 
measured. No measurements were able to be performed during the intervention phase and the follow-ups of skin measurements could not be continued for longer than 20 minutes.

Together, the above factors have affected the scope and validity of the findings on the physiological responses of CRMRF in patients to some extent. Nonetheless, like the normative study results, there has not been any sharp decline in the responses to the intervention over the follow-up phase. Hence, it is proposed that the effects would have been sustained significantly for more than 20 minutes providing a longer 'therapy window'. The reported effects from the current patient study are based on the convention that the participants maintained their treatment position throughout the experiment (lying in supine).

\section{Conclusions}

The moderately thermal CRMRF delivered to participants affected by OA knee yielded highly significant gains in SKT, SBF, deep blood flow volume and intensity of flow over the placebo and control interventions. There was no meaningful change in blood flow velocity. Core temperature, BP and PR did not change significantly at any time in any group. The findings were generally more pronounced than those obtained from the normative sample in the previous laboratory studies; however, the patients had received a higher average dose of CRMRF (hence a higher amount of energy) compared to those in the normative study. The whole-body SAR was not significantly different between the two samples. The results confirm that the physiological effects of CRMRF in an asymptomatic population can be reproduced in a patient population potentially in a more pronounced manner, and per se the effects may indicate the presence of clinical benefits such as those already reported. However, owing to differences in RF doses used in the two studies further research is needed to better understand the differences in physiological responses between patients and asymptomatic people. 


\section{Acknowledgements}

The authors would like to thank all the patients and the clinical and administrative staff at the Safari Therapy Unit, Hemel Hempstead Hospital for helping with this study. The authors acknowledge Indiba S. A., Barcelona, Spain for funding this study.

\section{Author contributions}

The first author $(\mathrm{BK})$ is responsible for the acquisition and analysis of data and writing up this manuscript. The second author (TW) is responsible for the critical revision of this manuscript and the conception and overall supervision of this research project. Both authors are responsible for the design of the study. Both authors have approved the final version of this manuscript and agree to be accountable for all aspects of the work, its accuracy and integrity. The authors also confirm that all persons designated as authors qualify for authorship, and all those who qualify for authorship are listed.

\section{Declaration of interest}

The University of Hertfordshire are in receipt of an industry-linked research funding related to this programme of research from Indiba S. A. (Barcelona, Spain). The industry funders had no role in the study design, data collection, and data analysis or in the preparation of this manuscript.

\section{References}

Adair, E. R. (1995). Thermal physiology of radiofrequency radiation (RFR) interactions in animals and humans. Radiofrequency Radiation Standards; Biological Effects, Dosimetry, Epidemiology and Public Health Policy, 245-269.

Adair, E. R., \& Black, D. R. (2003). Thermoregulatory responses to RF energy absorption. Bioelectromagnetics, Suppl 6, S17-38.

Al-Mandeel, M., M. (2004). Pulsed Shortwave Therapy: Its clinical use and physiological effects in healthy and osteoarthritic patients. Unpublished PhD thesis, University of Hertfordshire, Hertfordshire, UK.

Al-Mandeel, M. M., \& Watson, T. (2010). The thermal and nonthermal effects of high and low doses of pulsed short wave therapy (PSWT). Physiother Res Int, 15(4), 199-211. 
Altman, R., Asch, E., Bloch, D., Bole, G., Borenstein, D., Brandt, K., et al. (1986). Development of criteria for the classification and reporting of osteoarthritis. Classification of osteoarthritis of the knee. Diagnostic and Therapeutic Criteria Committee of the American Rheumatism Association. Arthritis Rheum, 29(8), 1039-1049.

Bricknell, R., \& Watson, T. (1995). The thermal effects of pulsed shortwave therapy. BJTR, 2, 430434.

Challis, L. J. (2005). Mechanisms for interaction between RF fields and biological tissue. Bioelectromagnetics, Suppl 7, S98-S106.

Draper, D. O., Castro, J. L., Feland, B., Schulthies, S., \& Eggett, D. (2004). Shortwave Diathermy and Prolonged Stretching Increase Hamstring Flexibility More Than Prolonged Stretching Alone. Journal of Orthopaedic and Sports Physical Therapy, 34(1), 13-20.

Draper, D. O., Knight, K., Fujiwara, T., \& Castel, J. C. (1999). Temperature change in human muscle during and after pulsed short-wave diathermy. J Orthop Sports Phys Ther, 29(1), 13-22.

Drust, B., Atkinson, G., Gregson, W., French, D., \& Binningsley, D. (2003). The effects of massage on intra muscular temperature in the vastus lateralis in humans. Int J Sports Med, 24(6), 395399.

Foster, K. R., \& Glaser, R. (2007). Thermal mechanisms of interaction of radiofrequency energy with biological systems with relevance to exposure guidelines. Health physics, 92(6), 609-620.

Foster, K. R., \& Morrissey, J. J. (2011). Thermal aspects of exposure to radiofrequency energy: report of a workshop. International journal of hyperthermia : the official journal of European Society for Hyperthermic Oncology, North American Hyperthermia Group, 27(4), 307-319.

Guirro, E. C., Guirro, R. R., Dibai-Filho, A. V., Pascote, S. C., \& Rodrigues-Bigaton, D. (2015). Immediate effects of electrical stimulation, diathermy, and physical exercise on lower limb arterial blood flow in diabetic women with peripheral arterial disease: a randomized crossover trial. J Manipulative Physiol Ther, 38(3), 195-202.

Jackson, A. S., \& Pollock, M. L. (1978). Generalized equations for predicting body density of men. $\mathrm{Br} J$ Nutr, 40(3), 497-504.

Jauchem, J. R. (2008). Effects of low-level radio-frequency ( $3 \mathrm{kHz}$ to $300 \mathrm{GHz}$ ) energy on human cardiovascular, reproductive, immune, and other systems: a review of the recent literature. Int J Hyg Environ Health, 211(1-2), 1-29.

Kumaran, B., Herbland, A., \& Watson, T. (2017). Continuous-mode 448 kHz capacitive resistive monopolar radiofrequency induces greater deep blood flow changes compared to pulsed mode shortwave: a crossover study in healthy adults. Eur J Physiother, 19(3), 137-146.

Kumaran, B., \& Watson, T. (2015). Thermal build-up, decay and retention responses to local therapeutic application of $448 \mathrm{kHz}$ capacitive resistive monopolar radiofrequency: A prospective randomised crossover study in healthy adults. Int J Hyperthermia, 31(8), 883895.

Kumaran, B., \& Watson, T. (2018). Skin thermophysiological effects of $448 \mathrm{kHz}$ capacitive resistive monopolar radiofrequency in healthy adults: A randomised crossover study and comparison with pulsed shortwave therapy. Electromagn Biol Med, 37(1), 1-12.

Kumaran, B., \& Watson, T. (2019). Treatment using $448 \mathrm{kHz}$ capacitive resistive monopolar radiofrequency improves pain and function in patients with osteoarthritis of the knee joint: a randomised controlled trial. Physiotherapy, 105(1), 98-107.

Laufer, Y., \& Dar, G. (2012). Effectiveness of thermal and athermal short-wave diathermy for the management of knee osteoarthritis: a systematic review and meta-analysis. Osteoarthritis Cartilage, 20(9), 957-966.

Mandryk, R. L., \& Atkins, M. S. (2007). A fuzzy physiological approach for continuously modeling emotion during interaction with play technologies. International journal of human-computer studies, 65(4), 329-347. 
Mandryk, R. L., Inkpen, K. M., \& Calvert, T. W. (2006). Using psychophysiological techniques to measure user experience with entertainment technologies. Behaviour \& Information Technology, 25(2), 141-158.

Noe, F., Amarantini, D., \& Paillard, T. (2009). How experienced alpine-skiers cope with restrictions of ankle degrees-of-freedom when wearing ski-boots in postural exercises. J Electromyogr Kinesiol, 19(2), 341-346.

Petrofsky, J. S., Laymon, M., \& Lee, H. (2013). Effect of heat and cold on tendon flexibility and force to flex the human knee. Med Sci Monit, 19, 661-667.

Robertson, V. J., Ward, A. R., \& Jung, P. (2005). The effect of heat on tissue extensibility: A comparison of deep and superficial heating. Archives of Physical Medicine and Rehabilitation, 86(4), 819-825.

Silverman, D. R., \& Pendleton, L. (1968). A comparison of the effects of continuous and pulsed shortwave diathermy on peripheral circulation. Arch Phys Med Rehabil, 49(8), 429-436.

Teslim, O. A., Adebowale, A. C., Ojoawo, A. O., Sunday, O. A., \& Bosede, A. (2013). Comparative effects of pulsed and continuous short wave diathermy on pain and selected physiological parameters among subjects with chronic knee osteoarthritis. Technology and Health Care, 21(5), 433-440.

Valtonen, E. J., Lilius, H. G., \& Svinhufvud, U. (1973). Effects of three modes of application of short wave diathermy on the cutaneous temperature of the legs. Eura Medicophys, 9(2), 49-52.

Wang, H., Zhang, C., Gao, C., Zhu, S., Yang, L., Wei, Q., et al. (2017). Effects of short-wave therapy in patients with knee osteoarthritis: a systematic review and meta-analysis. Clin Rehabil, 31(5), 660-671.

Yilmaz Kaysin, M., Akpinar, P., Aktas, I., Unlu Ozkan, F., Silte Karamanlioglu, D., Cagliyan Hartevioglu, H., et al. (2018). Effectiveness of Shortwave Diathermy for Subacromial Impingement Syndrome and Value of Night Pain for Patient Selection: A Double-Blinded, Randomized, Placebo-Controlled Trial. Am J Phys Med Rehabil, 97(3), 178-186. 
Table 1: Demographic and mean (SD) anthropometric data from the 45 study participants

\begin{tabular}{|c|c|c|c|c|c|c|c|c|}
\hline \multirow[b]{2}{*}{ Study Group } & \multicolumn{4}{|c|}{ Demographic data } & \multicolumn{4}{|c|}{ Mean (SD) anthropometric data } \\
\hline & $\begin{array}{l}\text { Mean (SD) age } \\
\text { (years) }\end{array}$ & Males & Females & $\begin{array}{l}\text { Mean (SD) OA } \\
\text { duration (years) }\end{array}$ & Height (m) & Weight (kg) & Body fat (\%) & BMI \\
\hline CRMRF & $63(10)$ & 6 & 9 & $5.6(4.1)$ & $1.67(0.10)$ & $88(17)$ & $39(7.6)$ & $31(3.8)$ \\
\hline CRMRF placebo & $63(10)$ & 6 & 9 & $4.3(3.9)$ & $1.63(0.09)$ & 82 (17) & $38(13)$ & $31(5.6)$ \\
\hline Control & $60(6.2)$ & 6 & 9 & $6.0(5.7)$ & $1.67(0.07)$ & $96(16)$ & $42(8.5)$ & $34(5.7)$ \\
\hline
\end{tabular}


Table 2: ANOVA main results for all outcomes.

\begin{tabular}{|c|c|c|c|c|c|c|}
\hline \multirow{2}{*}{$\begin{array}{l}\text { Outcome } \\
\text { measure }\end{array}$} & \multicolumn{3}{|c|}{ Within group change (main effect for time) } & \multicolumn{3}{|c|}{ Interaction between group and time } \\
\hline & F value & $\begin{array}{l}\text { Degrees of } \\
\text { freedom }\end{array}$ & $p$ value* & $F$ value & $\begin{array}{l}\text { Degrees of } \\
\text { freedom }\end{array}$ & $p$ value* \\
\hline SKT & 155.643 & $1.377,57.817$ & $<0.001$ & 238.511 & 2.753 & $<0.001$ \\
\hline SBF & 21.793 & $1.554,65.285$ & $<0.001$ & 45.058 & 3.109 & $<0.001$ \\
\hline $\begin{array}{l}\text { Blood } \\
\text { flow } \\
\text { volume }\end{array}$ & 57.081 & 1,42 & $<0.001$ & 75.930 & 2 & $<0.001$ \\
\hline $\begin{array}{l}\text { Blood } \\
\text { flow } \\
\text { intensity }\end{array}$ & 25.578 & 1,42 & $<0.001$ & 43.744 & 2 & $<0.001$ \\
\hline $\begin{array}{l}\text { Blood } \\
\text { flow } \\
\text { velocity }\end{array}$ & 0.129 & 1,42 & & 3.976 & 2 & 0.026 \\
\hline
\end{tabular}

* The $p$ values are reported only where the interactions were statistically significant at $p \leq 0.05$ and were meaningful. 
Table 3: Within-group comparisons from the three study groups on the five outcome measures

\begin{tabular}{|c|c|c|c|c|c|c|c|}
\hline \multirow{2}{*}{$\begin{array}{l}\text { Outcome } \\
\text { measure }\end{array}$} & \multirow[b]{2}{*}{ Group } & \multicolumn{3}{|c|}{ Baseline to post treatment } & \multicolumn{3}{|c|}{ Baseline to 20-minute follow-up } \\
\hline & & $\begin{array}{l}\text { Mean } \\
\text { difference }\end{array}$ & $p$ value* & $\begin{array}{l}\text { Effect size** } \\
\text { (Hedges's g) }\end{array}$ & $\begin{array}{l}\text { Mean } \\
\text { difference }\end{array}$ & $p$ value* & $\begin{array}{l}\text { Effect size** } \\
\text { (Hedges's g) }\end{array}$ \\
\hline SKT & $\begin{array}{l}\text { CRMRF } \\
\text { CRMRF placebo } \\
\text { Control }\end{array}$ & $\begin{array}{l}-0.342 \\
0.204 \\
-0.045\end{array}$ & $\begin{array}{l}<0.001 \\
0.003\end{array}$ & $\begin{array}{l}5.99 \\
0.48\end{array}$ & $\begin{array}{l}-0.307 \\
0.035 \\
-0.115\end{array}$ & $\begin{array}{l}<0.001 \\
0.09 \\
<0.001\end{array}$ & $\begin{array}{l}5.46 \\
1.06\end{array}$ \\
\hline SBF & $\begin{array}{l}\text { CRMRF } \\
\text { CRMRF placebo } \\
\text { Control }\end{array}$ & $\begin{array}{l}-0.766 \\
0.135 \\
0.029\end{array}$ & $<0.001$ & 2.81 & $\begin{array}{l}-0.540 \\
0.123 \\
0.016\end{array}$ & $<0.001$ & 2.26 \\
\hline $\begin{array}{l}\text { Blood } \\
\text { flow } \\
\text { volume }\end{array}$ & $\begin{array}{l}\text { CRMRF } \\
\text { CRMRF placebo } \\
\text { Control }\end{array}$ & $\begin{array}{l}-0.647 \\
0.067 \\
-0.009\end{array}$ & $<0.001$ & 1.75 & & & \\
\hline $\begin{array}{l}\text { Blood } \\
\text { flow } \\
\text { intensity }\end{array}$ & $\begin{array}{l}\text { CRMRF } \\
\text { CRMRF placebo } \\
\text { Control }\end{array}$ & $\begin{array}{l}-2.611 \\
0.131 \\
0.311\end{array}$ & $<0.001$ & 2.17 & & & \\
\hline $\begin{array}{l}\text { Blood } \\
\text { flow } \\
\text { velocity }\end{array}$ & $\begin{array}{l}\text { CRMRF } \\
\text { CRMRF placebo } \\
\text { Control }\end{array}$ & $\begin{array}{l}-0.591 \\
0.521 \\
0.251\end{array}$ & 0.049 & 0.54 & & & \\
\hline
\end{tabular}

* The $p$ values are reported only where the interactions were statistically significant at $p \leq 0.05$ and were meaningful. Adjustment for multiple comparison: Bonferroni.

** Effect sizes are reported only where the interactions were statistically significant at $p \leq 0.05$ and were meaningful. 
Table 4: Between-group comparisons from the three study groups on the five outcome measures

\begin{tabular}{|c|c|c|c|c|c|c|c|c|c|c|}
\hline \multirow{2}{*}{$\begin{array}{l}\text { Outcome } \\
\text { measure }\end{array}$} & \multirow[b]{2}{*}{ Time points } & \multicolumn{3}{|c|}{ CRMRF versus CRMRF placebo } & \multicolumn{3}{|c|}{ CRMRF versus control } & \multicolumn{3}{|c|}{ CRMRF placebo versus control } \\
\hline & & $\begin{array}{l}\text { Mean } \\
\text { difference }\end{array}$ & $p$ value* & $\begin{array}{l}\text { Effect size** } \\
\text { (Hedges's g) }\end{array}$ & $\begin{array}{l}\text { Mean } \\
\text { difference }\end{array}$ & $p$ value* & $\begin{array}{l}\text { Effect size** } \\
\text { (Hedges's g) }\end{array}$ & $\begin{array}{l}\text { Mean } \\
\text { difference }\end{array}$ & $p$ value* & $\begin{array}{l}\text { Effect size** } \\
\text { (Hedges's g) }\end{array}$ \\
\hline \multirow[t]{2}{*}{ SKT } & Post treatment & 0.536 & $<0.001$ & 4.91 & 0.294 & $<0.001$ & 3.88 & -0.242 & $<0.001$ & 1.98 \\
\hline & 20-min follow-up & 0.331 & $<0.001$ & 2.87 & 0.191 & $<0.001$ & 2.16 & -0.140 & 0.003 & 1.04 \\
\hline \multirow[t]{2}{*}{ SBF } & Post treatment & 0.923 & $<0.001$ & 3.00 & 0.767 & $<0.001$ & 2.63 & -0.155 & & \\
\hline & 20-min follow-up & 0.685 & $<0.001$ & 2.50 & 0.529 & $<0.001$ & 1.98 & -0.156 & & \\
\hline $\begin{array}{l}\text { Blood } \\
\text { flow } \\
\text { volume }\end{array}$ & Post treatment & 0.690 & $<0.001$ & 1.88 & 0.659 & $<0.001$ & 1.89 & -0.031 & & \\
\hline $\begin{array}{l}\text { Blood } \\
\text { flow } \\
\text { intensity }\end{array}$ & Post treatment & 2.462 & $<0.001$ & 2.05 & 2.718 & $<0.001$ & 2.34 & 0.257 & & \\
\hline $\begin{array}{l}\text { Blood } \\
\text { flow } \\
\text { velocity }\end{array}$ & Post treatment & 1.156 & 0.025 & 0.99 & 0.675 & & & -0.482 & & \\
\hline
\end{tabular}

* The $p$ values are reported only where the interactions were statistically significant at $p \leq 0.05$ and were meaningful. Adjustment for multiple comparison: Bonferroni.

** Effect sizes are reported only where the interactions were statistically significant at $p \leq 0.05$ and were meaningful. 
Figure 1: The experimental timeline.

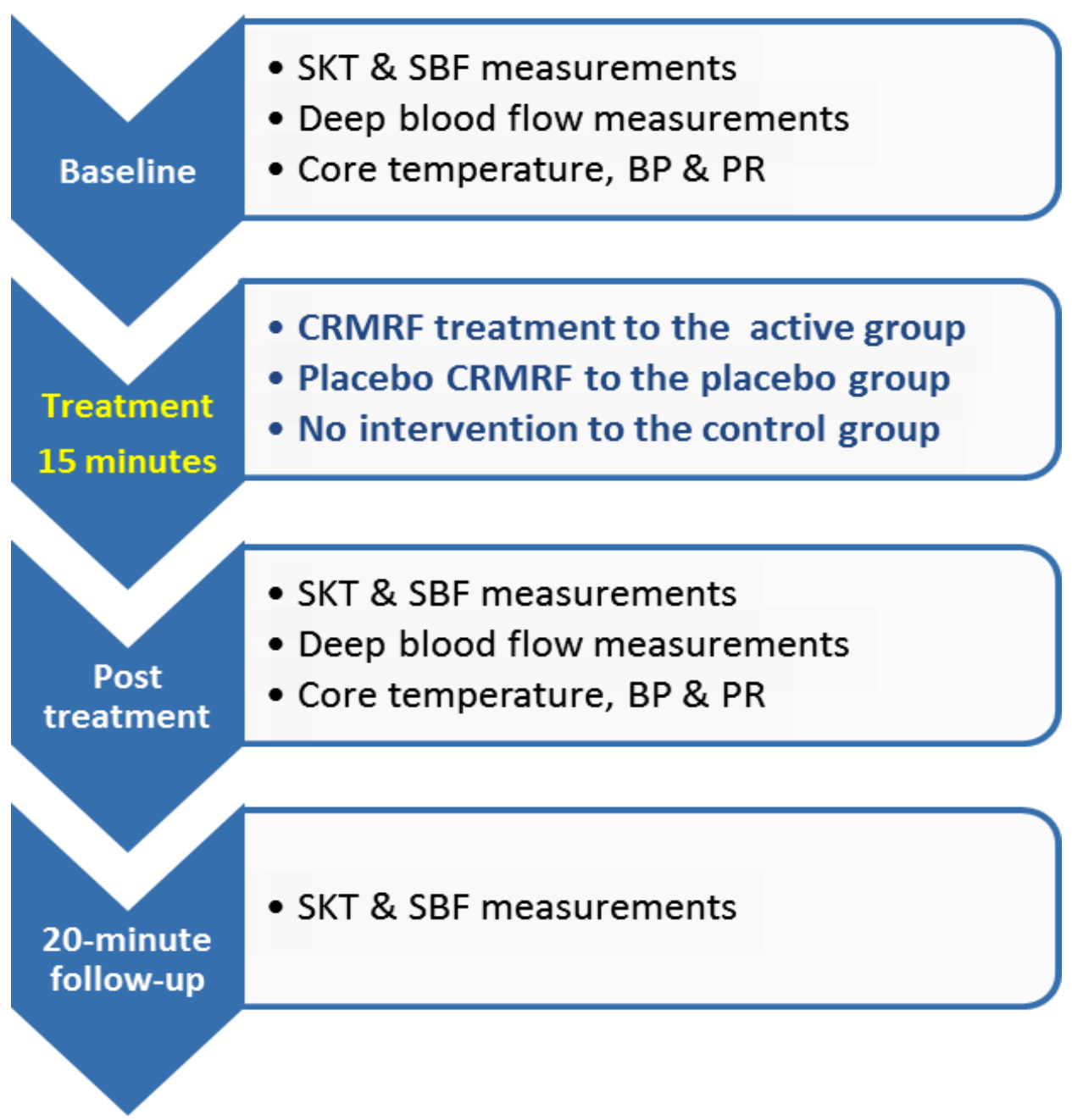


Figure 2: The sample data obtained from one participant in the CRMRF group.
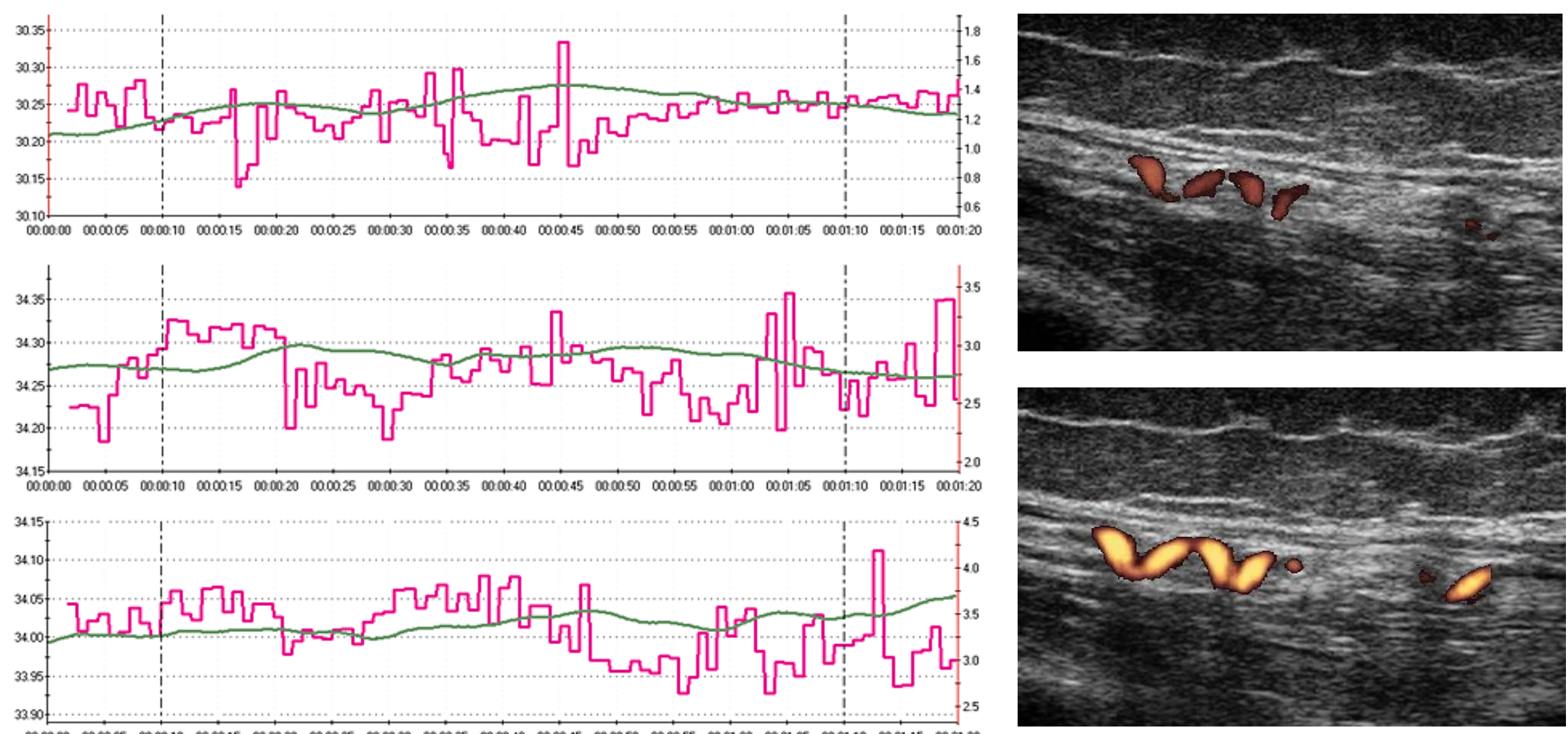

Figure legend:

Left: Sample images showing pre, post and follow-up (top to bottom) skin measurements (the green line denotes SKT in degree Celsius and the purple line denotes SBF in arbitrary units). Right: pre and post (top to bottom) deep blood flow volume. The vertical doted lines indicate one-minute intervals. 
Figure 3: The mean (SD) SKT recorded at the three time points from the three groups.
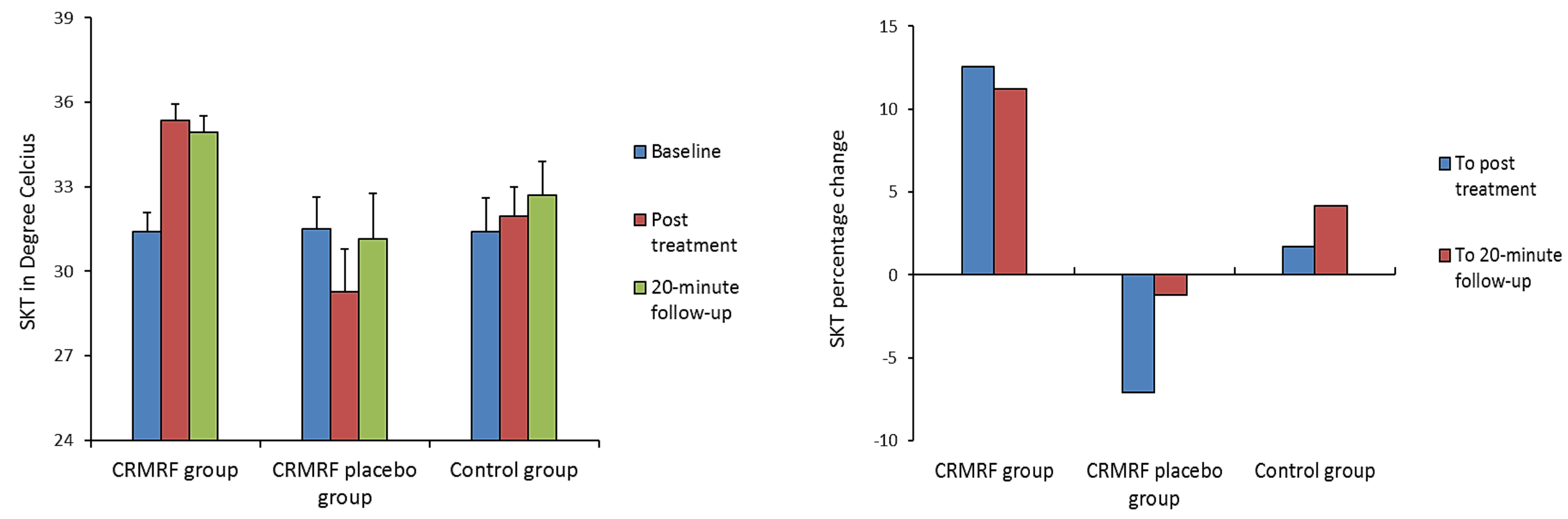
Figure 4: The mean (SD) SBF recorded at the three time points from the three groups.
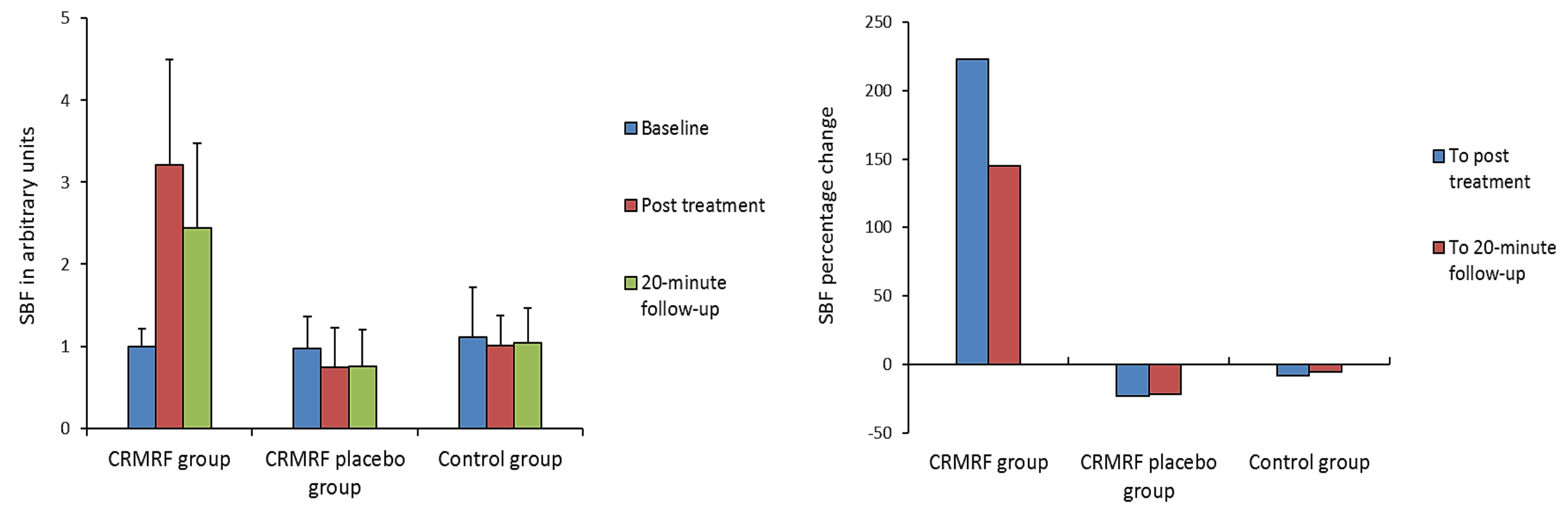
Figure 5: The mean (SD) deep blood flow volume at the two time points from the three groups.
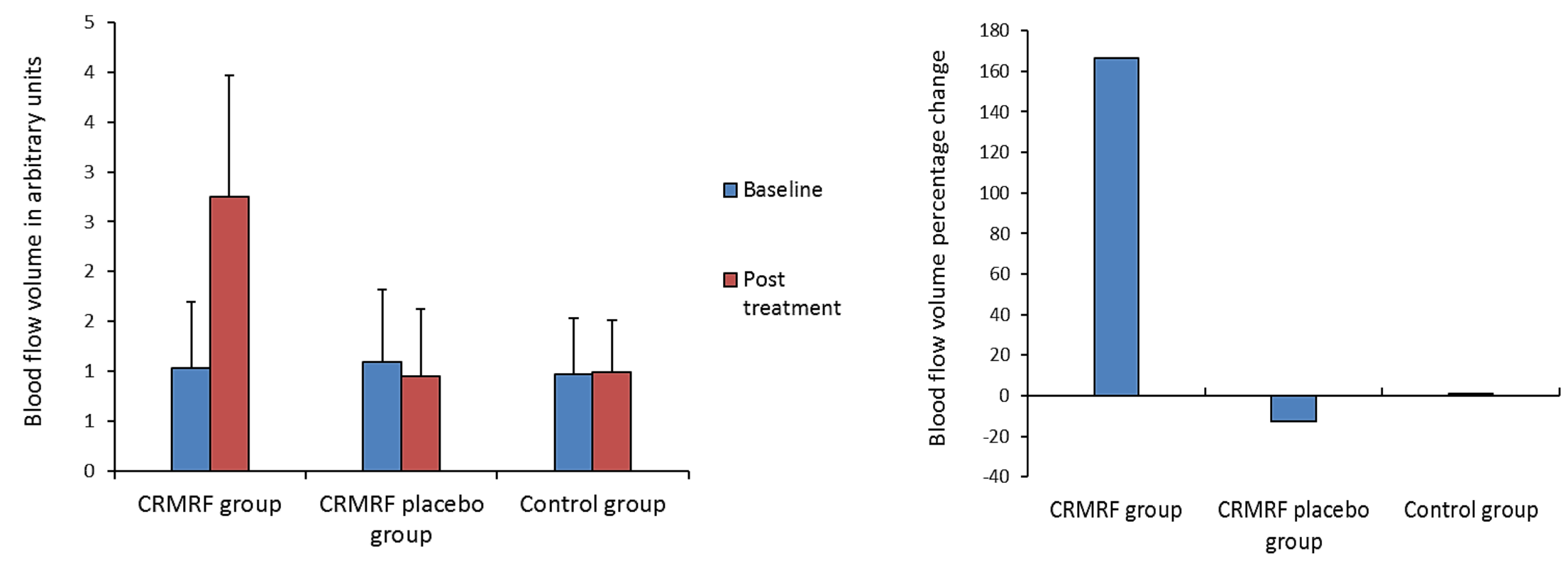
Figure 6: The mean (SD) intensity of blood flow at the two time points from the three groups.
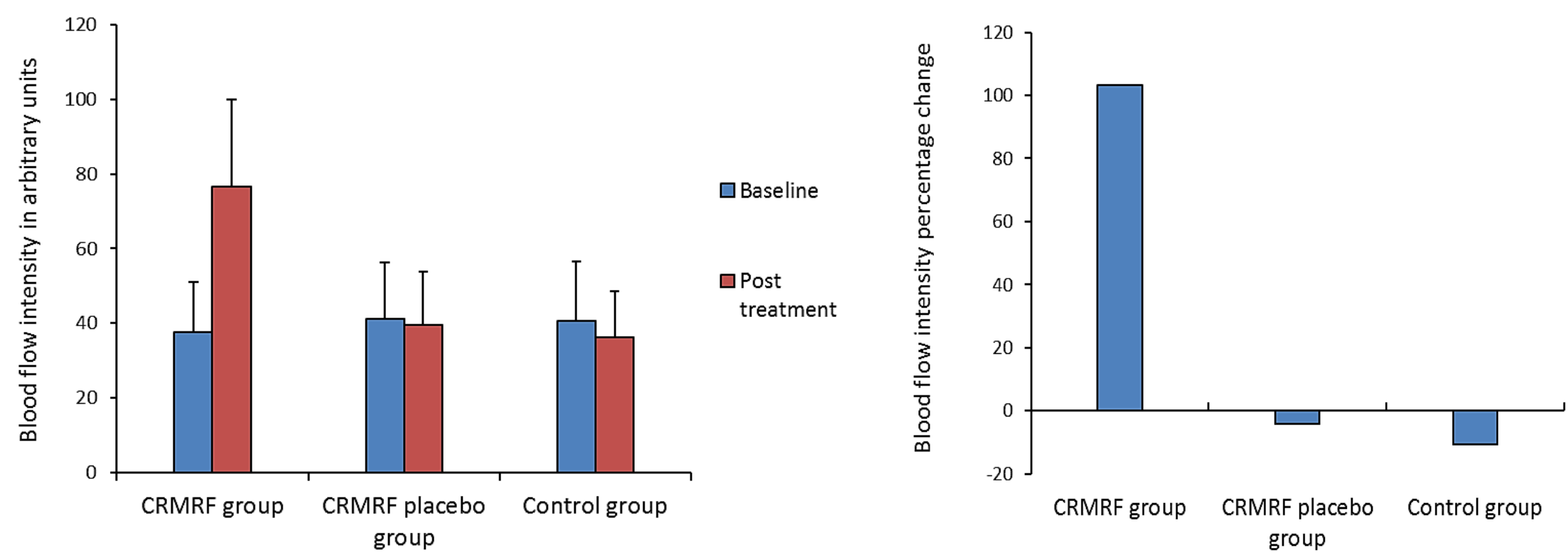
Figure 7: Comparison of skin physiological data between patients and asymptomatic adults.
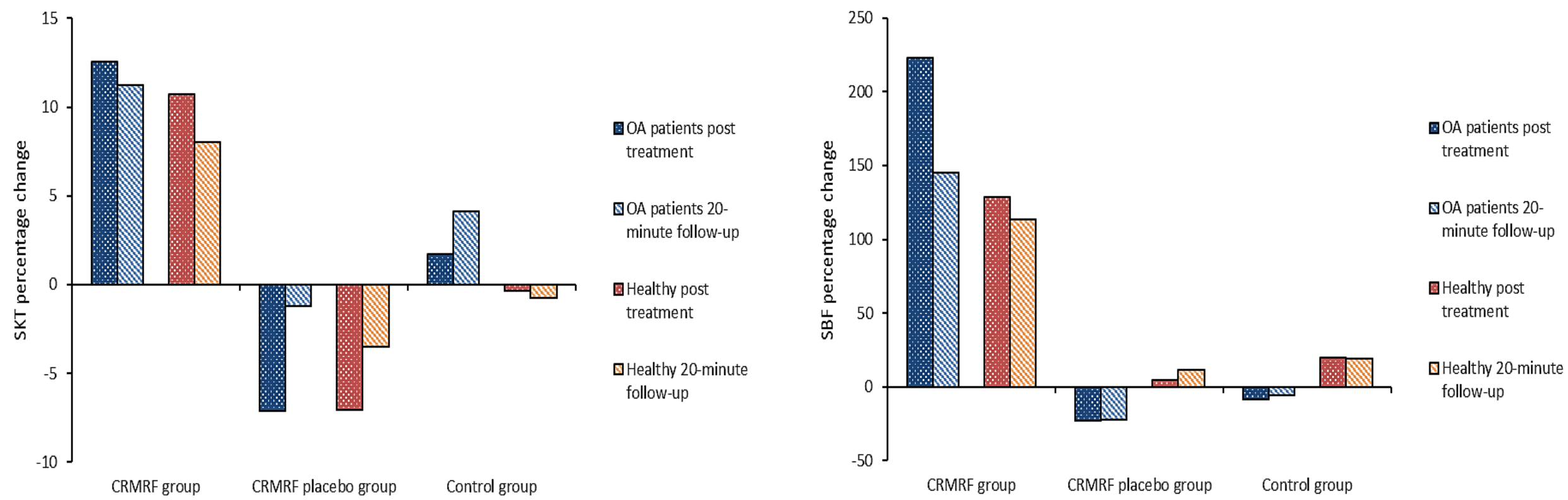
Figure 8: Comparison of deep blood flow data between patients and asymptomatic adults.
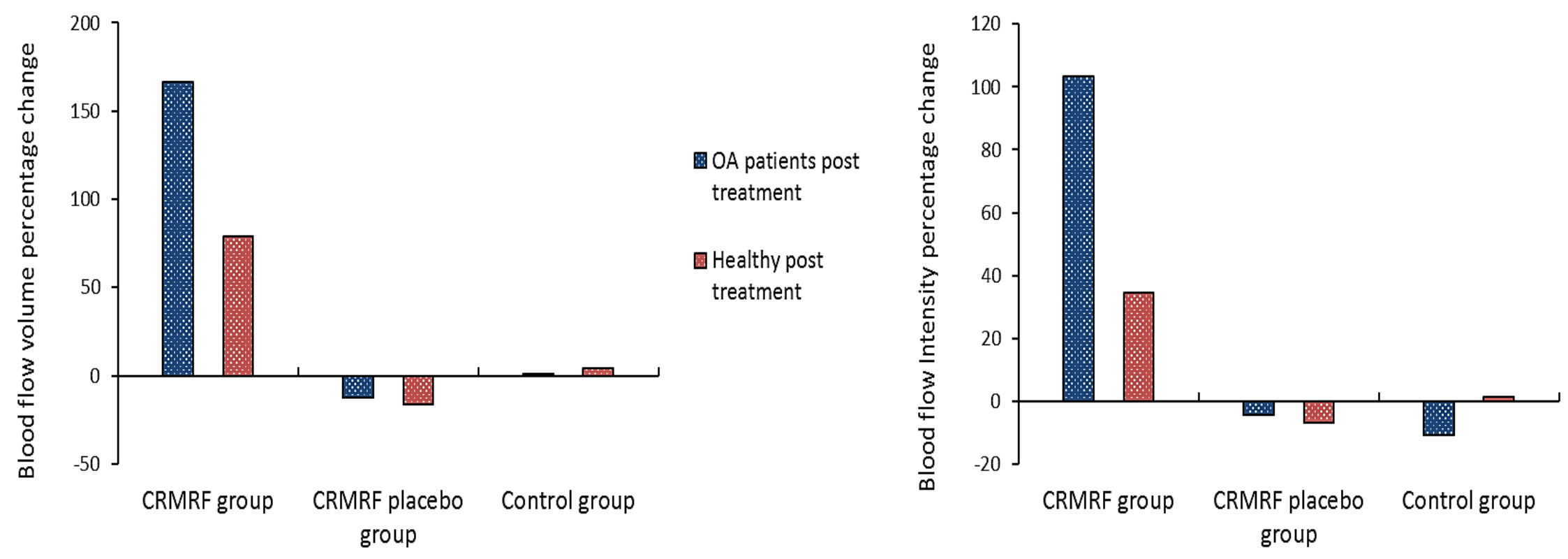

OA patients post treatment

- Healthy post treatment 